\title{
La vida a las puertas de la jaula de hierro. Relaciones de poder inherentes a las prácticas de participación en el Tercer Sector ${ }^{1}$
}

\section{The life at the gates of the iron cage. Power relations through practices of participation in the Third Sector}

Miguel Ángel Sahagún Padilla

Universitat Autònoma de Barcelona

miguelangel.sahagun@uab.cat

\begin{abstract}
Resumen
Abstract

En este texto describo a grandes rasgos $\mathrm{mi}$ In this work I offer an overview of my doctoral research investigación doctoral sobre formas de poder on forms of power that are inherent to participatory inherentes a las prácticas de participación en practices in Third Sector organizations. The research organizaciones del Tercer Sector. El planteamiento del problem has been placed within a framework that problema se enmarca en el interés por la imbricación intends to address the overlapping character of power entre fenómenos organizativos y fenómenos de poder. phenomena and organizational phenomena. The El marco conceptual se configura a partir del trabajo de theoretical framework draws from the works of Foucault Foucault (poder), Bourdieu (habitus, campo), Bachrach (power), Bourdieu (habitus, field), Bachrach and Baratz y Baratz (no-decisión) y Goffman (footing). El diseño (non-decision) and Goffman (footing). The method metodológico consiste en un estudio de tipo etnográfico consists of an ethnographic study which is focused on focalizado en la interpretación de prácticas. Los the interpretation of practices. Results are deployed in resultados se presentan en forma de tres grandes the form of three grand maps, each of which belongs to mapas que se corresponden con distintos planos a specific analytic layer: a) intersectorial exchanges analíticos: a) intercambios intersectoriales $y$ sus and their effects; b) organizational configuration and efectos, b) configuración y funcionamiento organizativo functioning and c) symbolic aspects. Conclusions y c) aspectos simbólicos. En las conclusiones se underline the neutralizing effects of Third Sector as a enfatizan los efectos neutralizadores del Tercer Sector space for participation.

como vía para la participación.
\end{abstract}

Palabras clave: Relaciones de poder; Participación; Keywords: Power relations; Participation; Third Sector; Tercer Sector; Prácticas sociales

Social practices

\section{Descripción de la tesis}

¿Por qué la participación es un tema ineludible en tantos ámbitos de la vida social? ¿Qué es lo que hace de la participación una exigencia inexcusable en cierta modalidad de organizaciones? ¿Cuál es el papel

\footnotetext{
${ }^{1}$ Tesis doctoral dirigida por Francisco Elejabarrieta y Carmen Martínez Gonzalez. Departament de Psicologia Social, Universitat Autònoma de Barcelona. Disponible en: http://www.tesisenxarxa.net/TDX-0925109-134029/
} 
de la participación - de sus diversas concepciones y prácticas - en el entramado de acciones y relaciones que configuran a esas organizaciones?

El objeto de estudio de esta tesis se elaboró a partir de estas preguntas. En concreto, su delimitación fue producto de una mirada problematizadora de la confluencia entre: a) la participación, como idea y como práctica en los ámbitos más variados y bajo las concepciones más diversas; b) el Tercer Sector, como conjunto más o menos delimitado de organizaciones distinguibles respecto a los sectores público o privado y c) en el plano conceptual, el entrecruzamiento entre fenómenos organizativos y fenómenos de poder.

La investigación tuvo como objetivo analizar las prácticas de participación en organizaciones del Tercer Sector en función de los fenómenos de poder que son inherentes a esas prácticas, destacando el papel de la toma de decisiones y la centralidad de la participación como una característica del Tercer Sector que se considera obvia e indispensable.

La revisión de literatura sobre participación y Tercer Sector constituye la primera fase del trabajo realizado. El primer capítulo de la tesis ofrece una visión problematizadora de las concepciones y aproximaciones a la participación, enfatizando las diferencias entre las formas de entenderla y llevarla a la práctica, así como aquellos aspectos del fenómeno no tomados en cuenta y las premisas en las que se basa la producción existente. El segundo capítulo tiene como propósito describir y discutir el fenómeno del Tercer Sector, las formas en que ha sido concebido y sus relaciones con el Estado y con el Mercado como los grandes referentes con base en los cuales se define.

La segunda fase se centró en el desarrollo el marco conceptual en el que se sustentó el planteamiento del problema y el diseño de la investigación. El marco se organizó en tres niveles: a) la concepción de poder de Foucault (1976) en el nivel más amplio; b) los vínculos entre habitus y campo (Bourdieu, 1972; 1980) como lugar del que emergen las prácticas en tanto que mecanismo de reproducción social y, c) a un nivel más específico y próximo - la interacción -, los procesos de no decisión (Bachrach y Baratz, 1962; 1963; 1970) y la noción de footing formulada por Goffman (1981). El Capítulo 3 presenta la concepción de poder de Foucault contrastándola con otras concepciones de alcances más limitados y subrayando su capacidad para un abordaje analítico de las relaciones complejas entre participación y Tercer Sector. El Capítulo 4 presenta y discute los conceptos clave de la teoría de prácticas sociales de Bourdieu. El Capítulo 5 se centra en fenómenos específicos como la toma de decisiones y los encuentros sociales, que resultan de especial relevancia en la comprensión de la articulación entre participación y Tercer Sector.

La tercera fase del proceso se dedicó al planteamiento de los objetivos, al diseño del método, al trabajo de campo y al análisis. El Capítulo 6 hace una síntesis de los capítulos anteriores, resaltando las preguntas y las direcciones a seguir como base para los cuatro objetivos generales que sirven como ejes del estudio: a) describir exhaustivamente las prácticas de participación de organizaciones del Tercer Sector; b) analizar los procesos de toma de decisiones; c) analizar las prácticas de participación en función de las relaciones de poder que les son inherentes y d) delimitar las concepciones y evidencias que hacen posible pensar y hablar de la participación como un requisito natural u obvio del Tercer Sector. El Capítulo 7 detalla el método y el procedimiento seguidos: estudio de tipo etnográfico; 
muestreo teóricamente informado²; entrevistas semiestructuradas, observación y selección de documentos como técnicas de obtención de datos y análisis interpretativo de prácticas. El diseño del método tenía por objetivo clarificar los significados, condiciones de posibilidad y direcciones estratégicas de las prácticas de participación, así como las relaciones entre ellas, especialmente las relaciones de interferencia. En el plano más operativo, se incluyeron en el diseño algunos lineamientos de la Grounded Theory (Strauss y Corbin, 1998). En cuanto a la información analizada, se trabajó con un corpus conformado por a) entrevistas semi-estructuradas con personas involucradas en las organizaciones incluidas en la muestra, b) observación y registro de eventos cotidianos y especiales en las mismas organizaciones y c) documentos seleccionados en función de su relación con lo observado o con lo conversado durante las entrevistas; es decir, en función de su papel en las prácticas a analizar. El capítulo enfatiza la centralidad de la noción de prácticas sociales, destacando sus implicaciones como unidad de análisis.

En cuanto a los resultados, los capítulos $8,9,10$ y 11 presentan tres grandes mapas que abordan el fenómeno de estudio en planos diferentes. El Capítulo 8 ofrece una introducción a los mapas, resaltando su carácter distintivo, estructura y contenidos. El Capítulo 9 presenta un mapa 'económico' que parte de la idea de las actividades de participación como bienes intercambiables cuya producción y circulación condiciona el funcionamiento de las organizaciones del Tercer Sector. El Capítulo 10 presente un mapa 'físico' dedicado al proceso de conformación y al funcionamiento de las organizaciones del Tercer Sector en tanto que espacio híbrido que procedimentaliza y fagocita ciertas formas de acción colectiva. El Capítulo 11, un mapa 'meteorológico', está dedicado a los aspectos simbólicos de la actividad del Tercer Sector, resaltando la noción de lo biempensante - la forma en que configura temas relevantes en una modalidad de no-discusión - y el papel de los lugares comunes o iconos del Tercer Sector (voluntariado, poder, eficacia y eficiencia, etcétera).

El Capítulo 12 presenta las conclusiones de la tesis. Los resultados expuestos en los mapas se retoman de forma sintética como base para señalar las conclusiones del estudio: a) un Tercer Sector cuyos efectos formalizadores neutralizan el potencial transformador de la actividad colectiva; b) una participación atrapada en el procedimiento y c) la dificultad de convertir en tema de discusiones la complicidad no deliberada de los agentes cuya actividad sostiene el Tercer Sector con sus efectos políticamente neutralizadores.

\section{Referencias}

Bachrach, P. y Baratz, M.S. (1962). Two faces of power. The American Political Science Review, 56(4). $947-952$.

Bachrach, P. y Baratz, M.S. (1963). Decisions and Non-decisions: An analitical framework. The American Political Science Review, 57(3). 632 - 642.

\footnotetext{
${ }^{2}$ La estrategia de muestreo fue producto de un híbrido entre el muestreo por conveniencia y las características principales del muestreo 'bola de nieve'. La muestra estuvo integrada por cuatro tipos de organización en función del cruce de dos dimensiones: a) carácter paradigmático respecto a los criterios que distinguen a las organizaciones del Tercer Sector de las organizaciones Públicas o Privadas (organizaciones paradigmáticas o liminares) y b) implicación en asuntos de interés público con gran resonancia en su contexto (organizaciones implicadas o no implicadas).
} 
Bachrach, P. y Baratz, M.S. (1970). Power and poverty. Theory and practice. New York: Oxford University Press.

Bourdieu, P. (1972). Outline of a theory of practice. New York: Cambridge University Press; 1977.

Bourdieu, P. (1980). El sentido práctico. Madrid: Taurus; 1991.

Foucault, M. (1976). Historia de la sexualidad: la voluntad del saber. Buenos Aires: Siglo XXI Editores; 1977.

Goffman, E. (1981). Forms of talk. Philadelphia; University of Pennsylvania Press.

Strauss, A.L. y Corbin, J. (1998). Basics of qualitative research: Techniques and procedures for developing grounded theory (2nd ed.). Thousand Oaks: Sage Publications.

\section{Formato de citación}

Sahagún, Miguel (2009). La vida a las puertas de la jaula de hierro. Relaciones de poder inherentes a las prácticas de participación en el Tercer Sector. Athenea Digital, 16, 181-184. Disponible en http://psicologiasocial.uab.es/athenea/index.php/atheneaDigital/article/view/682.

\begin{tabular}{l}
\hline Este texto está protegido por una licencia Creative Commons. \\
Usted es libre de copiar, distribuir y comunicar públicamente la obra bajo las siguientes condiciones: \\
Reconocimiento: Debe reconocer y citar al autor original. \\
No comercial. No puede utilizar esta obra para fines comerciales. \\
Sin obras derivadas. No se puede alterar, transformar, o generar una obra derivada a partir de esta obra. \\
$\underline{\text { Resumen de licencia }-\underline{\text { Texto completo de la licencia }}}$ \\
\end{tabular}

